\title{
OBSERVATIONS ON THE TENDENCY OF THE DIPH- THERIA BACILLUS TO LOCALIZE IN THE UPPER RESPIRATORY TRACT
}

\author{
THE IMPORTANCE OF THIS FACT IN ROUTINE CULTURE WORK *
}

\author{
DAMON ORIAN WALTHALL, B.S. \\ ANN ARBOR, MICH.
}

Because of past experiences in finding that the organisms of diphtheria may be present in the larynx and absent in cultures taken from the nose and throat, or positive in the nose and absent in the throat, and so on, at the suggestion of Dr. Cowie I have made a number of observations with this point in view.

Of the eight cases which I wish to report, the organisms confined themselves to one locality in five cases, which I classify as Group 1; to more than one locality in three cases, which I classify as Group 2.

\section{GROUP 1}

CASE 1.- Ione O., aged 25, with marked clinical unitonsillar diphtheria, entered the hospital Feb. 8, 1916. Diphtheria organisms were present in every culture taken from the right tonsil, absent from every culture taken from the left tonsil and pillars, the pharyngeal wall and lateral bands and from the nose. A total of thirty-three cultures, covering a period of twelve days, was taken. Antitoxin, 10,000 units, was administered intravenously, and 10,000 units intramuscularly.

On March 3, 1916, the patient returned, complaining of slight epistaxis, but a nose culture was negative.

CASE 2.-Luella S., aged 21, with marked clinical diphtheria of the lateral bands, entered the hospital Jan. 25, 1916. General cultures taken January 26 and 29 gave positive findings. Special cultures were begun February 1. The organisms were at all times present in cultures taken from the pharyngeal wall and lateral bands, but were not found in cultures taken from the tonsils and pillars or in those from the nose. A total of twenty-two cultures was taken, covering a period of twenty days. Antitoxin was administered, 10,000 units intramuscularly, 10,000 units intravenously.

CASE 3.-Ruth W., aged 21 , a diphtheria carrier, entered the contagious hospital Dec. 2, 1915, having suffered with nose bleed two weeks before. General throat culture was negative; nasal culture positive. Nose and throat cultures taken simultaneously showed in each instance organisms in those taken from the nose, but not at any time in those taken from the throat. A total of thirty cultures, covering a period of twenty days, was made. The patient was discharged from the hospital after three negative cultures, the last one being taken Dec. 22, 1915.

* Submitted for publication April 27, 1916.

* From Department of Pediatrics and Contagious Diseases, University of Michigan, Ann Arbor. 
March 18, 1916, she returned to the hospital, again showing positive general throat cultures. Special cultures were again instituted, and it was found that a positive culture was in each instance obtained from the pharyngeal wall and lateral bands, but cultures from the tonsillar fossae and from the nose were negative. (This patient had had adenoid and tonsil operation previous to her first entrance to the hospital.) Total cultures numbering twenty-five were taken, covering a period of fourteen days. The patient is still under observation. The Schick test is negative.

CaSe 4.-Ernest D., aged 23, entered Contagious Hospital Feb. 29, 1916, showing mild clinical laryngeal diphtheria. Organisms were present in cultures taken from the larynx, but absent each time in cultures taken from the tonsils and pillars and from the pharyngeal wall and lateral bands and nose. Total cultures numbering twenty-five were taken, covering a period of sixteen days. Antitoxin was administered intravenously, to the amout of 10,000 units.

CASE 5.-Marion Y., aged 20, entered the Contagious Hospital service Jan. 5, 1916, showing laryngeal diphtheria. General culture in this case taken January 1, in the beginning of the illness, was negative. The organisms were present in cultures taken directly from the larynx until January 4 . After this, cultures were taken from the tonsils and pillars, the pharyngeal wall, lateral bands and from the nose. They were in every instance negative. Total cultures numbering twenty-eight were taken, covering a period of thirty-one days. Antitoxin was administered, 10,000 units intravenously, 20,000 intramuscularly.

\section{GROUP 2}

Case 6.-John S., aged 2, a diphtheria carrier, entered the Contagious Hospital, Dec. 9, 1915. This patient had harelip and cleft palate, enlarged tonsils and a visible mass of adenoids. A general culture, made Feb. 13, 1916, was positive and continued so until March 16, when special cultures were begun. Positive cultures were now confined to the tonsils and the visible mass of adenoids. Cultures taken from the nasal mucous membranes were negative. Total cultures numbering twenty-four and covering a period of thirty-nine days were taken. The patient is still under observation.

CASE 7.--Harry S., aged 29, a diphtheria carrier, entered the hospital Feb. 16, 1916, with facial erysipelas; the throat cultures were negative. A general culture was positive on February 27 and continued to be so until March 13, when special cultures were started. The organisms were now found present in cultures taken from the pharyngeal wall and from the nasopharynx, taken per os, but they were not found in cultures taken from both tonsils, anterior nasal passages, both right and left, and from the external auditory canal. This patient had been operated on for mastoid disease a few days before entering the Contagious Hospital. Positive cultures were obtained from the mastoid wound on three different days. The patient is still under observation. Total cultures numbering thirty-eight were taken, covering a period of thirtyeight days. It may be of interest to note that a long organism was present in this case. It has been thought that these organisms are more virulent than the short ones.

CASE 8.-Sarah S., aged 4, on Dec. 22, 1915, showed clinical nasal diphtheria, which cleared up in a few days without any antitoxin. The patient was detained because of exposure to chickenpox before entering the hospital. General routine throat culture was positive Jan. 26, 1916. Special cultures were begun February 2. They were taken with a swab, those from the tonsils and pillars, pharyngeal wall and lateral bands proving to be negative. The tonsils in this case were so large that it was impossible to reach the pharynx with a swab without touching them. A stiff platinum loop was substituted for the swab February 11. The cultures were now positive from both tonsils and negative from the pharyngeal wall and nose. Total cultures numbering thirtythree were taken, covering a period of seventy days. 


\section{CONCLUSION}

1. All parts of the mucous membrane of the upper respiratory tract must be touched in making a satisfactory general culture.

2. The pharynx may be cultured and be negative for Klebs-Loeffler bacilli, but the organisms may be present in the nose or in the larynx and thus not be found except by special technic in culture work. Or as in Case 1, one tonsil may give positive cultures while all other portions of the mucous membranes may be negative. Nose bleed and hoarseness or loss of voice should attract attention to the nose or larynx.

3. Sterile cotton swabs are found to be most adaptable for getting culture material from all parts of the upper respiratory tract. Second in preference for culture taking is a platinum loop. The platinum wire must be quite heavy and stiff in order to prevent its curling and bending. It can be used only in taking cultures from tonsils, pillars, posterior pharyngeal wall and lateral bands. It cannot be used in taking nose and laryngeal cultures because of danger of trauma to the mucous membrane.

4. Several cases in this series show the necessity of having more than one or two cultures to determine that diphtheria organisms are absent from the upper respiratory tract. In this hospital at least three negative cultures are required before a patient may be discharged or before a nurse may return to the main hospital for service.

5. This interesting and peculiar tendency of the diphtheria organisms to localize in one part of the upper respiratory tract should be an important factor in deciding on surgical or medical treatment to eliminate the organisms from this part. 